\title{
Diamonds Utilized in the Development of Single Ion Detector with High Spatial Resolution
}

\author{
Shinobu Onoda, Takashi Yamamoto, Takeshi Ohshima, \\ Junichi Isoya*, Tokuyuki Teraji** and Kenji Watanabe** \\ Japan Atomic Energy Agency (JAEA), 1233 Watanuki, Takasaki, Gunma, 370-1292, Japan \\ * University of Tsukuba, 1-2 Kasuga, Tsukuba, Ibaraki 305-8550, Japan \\ ** National Institute for Materials Science (NIMS), 1-1 Namiki, Tsukuba, Ibaraki 305-0044, Japan \\ Fax: 81-27-346-9687, e-mail: onoda.shinobu@jaea.go.jp
}

\begin{abstract}
We are developing the Ion Photon Emission Microscopy (IPEM) system at JAEA. To observe a map by IPEM, the position where an ion strikes a scintillator placed over a microelectronics circuit is recorded together with the ion induced event. Since the spatial resolution is determined by the spot size of the Ion Beam Induced Luminescence (IBIL), the scintillator is one of the most important parts of IPEM. In this study we propose that a diamond containing a high concentration of Nitrogen Vacancy (NV) centers can be used as a scintillator with high spatial resolution. For both $\mathrm{Y}_{3} \mathrm{Al}_{5} \mathrm{O}_{12}$ : $\mathrm{Ce}(\mathrm{YAG}: \mathrm{Ce}$ ) and the diamond containing $\mathrm{NV}$ centers, the minimum spot size is a few micrometers. The IBIL intensity from the diamond containing NV centers is higher than that from YAG:Ce. According to these results, we suggest that a diamond containing NV centers is a rival candidate of a YAG:Ce from the point of view of single ion detection with high spatial resolution.
\end{abstract}

Key words: Diamond, NV center, Single Ion Detection, Ion Photon Emission Microscopy (IPEM)

\section{INTRODUCTION}

1.1 Ion Photon Emission Microscopy

It is well known that Single Event Effects (SEEs) cause microelectronic malfunctions and arise from the impact of high-energy heavy ion in radiation environments. Generally, SEEs are triggered by unexpected transient currents induced by the single ion strike. A focusedmicrobeam combined with a single ion hitting technique has a key advantage in evaluating the transient currents in specific device regions with a high spatial resolution. We have developed two systems to acquire two-dimensional maps of transient currents by using the focused microbeams connected with the $3 \mathrm{MV}$ tandem and Azimuthally Varying Field (AVF) cyclotron accelerators at Japan Atomic Energy Agency (JAEA), Takasaki [1-3]. These are called the Transient Ion Beam Induced Current (TIBIC) systems. The similar system called the Time Resolved Ion Beam Induced Current (TRIBIC) has been developed at Sandia National Laboratories (SNL) [4]. The powerful advantage of TIBIC mapping is the ability to determine the position dependence of the event induced by an ion. While the microbeam has many advantages for SEE testing, the transport and optimization of the microbeam requires much time and effort. Therefore, we are developing the new system which requires less effort.

A different way to perform mapping has been proposed by SNL and known as the Ion Photon Emission Microscopy (IPEM) [5-7]. To observe the map, it is not necessary to focus the beam at all. Instead of microbeam to control the position of the ion strike, a scintillator on a sample is used to record the position together with the ion induced event. The position signals are detected by a Position Sensitive Detector (PSD).
The scintillator is one of the most important parts of IPEM system. Recently Branson et al. have studied the optical properties of various scintillators [7]. They suggested that $\mathrm{Y}_{3} \mathrm{Al}_{5} \mathrm{O}_{12}: \mathrm{Ce}$ (YAG:Ce) is so far the best option for application in IPEM, because of its high emission rate, short decay time, and high radiation hardness. Additionally, the emission spectrum is particularly important. In the case of high energy ion irradiation in air, the gas molecules are ionized, the subsequent luminescence of air is detected as noise. Therefore, the emission wavelength of the scintillator should be different from that of air $(<450 \mathrm{~nm})$ [7]. Of course, the emission spectrum of the scintillator also has to match the sensitivity of the optical components and detectors.

\subsection{Optical Properties of Nitrogen Vacancy Center}

A variety of color centers in a diamond have been reported over last several decades [8]. The Nitrogen Vacancy (NV) center in a diamond is the most studied color centers, especially for Quantum Information Processing (QIP) applications, since it can act as a single photon source with a high emission rate even at room temperature [9]. The quantum efficiency of NV centers in a diamond, which is defined as the probability of emitting a photon once the system is prepared in the excited state, was estimated to be around 0.7 [9]. Furthermore, the count rate exceeds hundreds of thousands of counts per second. According to Photoluminescence (PL) measurements, there are two sharp emission peaks around 575 and $637 \mathrm{~nm}$ with pronounced and wide phonon side bands on the lower energy side of each peak, which is longer than the air luminescence as mentioned above. Based on these 


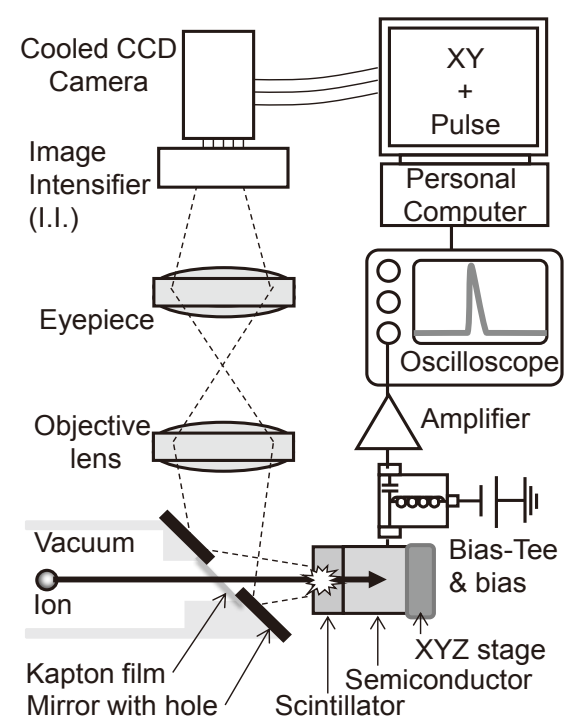

Fig. 1 Schematic diagram of the measurement system.

characteristics, we propose a new application of NV centers in a diamond as a scintillator for IPEM. In this paper, we compare the Ion Beam Induced Luminescence (IBIL) properties of diamonds with other scintillators.

\section{EXPERIMENTS}

2.1 Overview of IPEM setup at JAEA

Fig. 1 shows a schematic diagram of the measurement system developed at JAEA Takasaki. This system contains the following; (1) a beam extraction window (Kapton film, $7 \mu \mathrm{m}$ in thick) under a mirror with hole. The angle of hole is 45 degrees with a diameter of $1 \mathrm{~mm}$, and the mirror is mounted at an angle of 45 degrees to the objective lens.; (2) a scintillator and a semiconductor mounted on a micro XYZ stage; (3) an electronics for charge measurements including an amplifier, a bias-tee, a bias supply and an oscilloscope; and (4) a photon detection equipment including the microscope (Olympus, $\mathrm{BX} 51 \mathrm{M}$ ), the GaAsP Image Intensifier (I.I.) (Hamamatsu, C8600), and the cooled Charge Coupled Device (CCD) camera (Hamamatsu, C4880-50-26A). Fig. 2 (a) shows the Quantum Efficiencies (QEs) of I.I. and $\mathrm{CCD}$ as a function of the wavelength. Branson et al. have reported the PL spectrum of the commercial YAG:Ce as shown in Fig. 2 (b) [7]. As shown, the emission spectrum of YAG:Ce matches the sensitivities of the I.I. and the CCD.

The high energy ion beams accelerated by the AVF cyclotron are extracted from vacuum to air via the Kapton film. The extracted ion penetrates the scintillator on a semiconductor. The photons from the scintillator are detected by the cooled CCD camera. The position where the ion hit the scintillator can be calculated from the center of mass of ion induced luminescence. At the same time the ion induced transient current pulse generated at a semiconductor is recorded by the oscilloscope. Combining the position where ion hit and the transient current, a 2D map is observed.

\subsection{Samples}

Table I shows the scintillators used in this study. First scintillator is an inorganic scintillator, $\mathrm{ZnS}: \mathrm{Ag}(\# 1)$. The emission maximum, $\lambda_{\max }$ is at $450 \mathrm{~nm}$ [10]. The $\mathrm{ZnS}: \mathrm{Ag}$

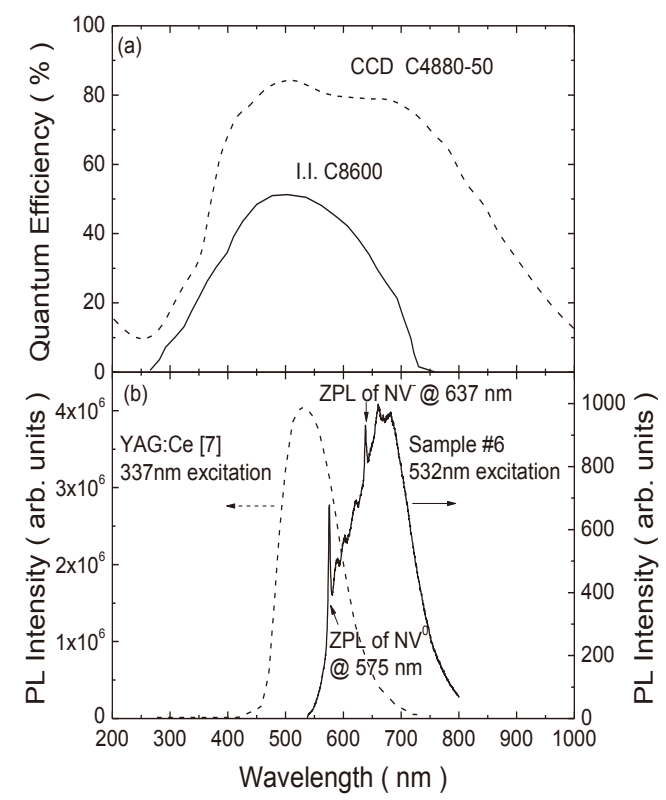

Fig. 2 QEs of I.I. and cooled CCD as a function of wavelength (a). The PL spectra of YAG:Ce excited by $337 \mathrm{~nm}$ laser [7] and diamond containing NV centers (\#6) excited by $532 \mathrm{~nm}$ laser at room temperature (b).

Table I. Overview of the scintillators studied

\begin{tabular}{|c|l|l|}
\hline$\#$ & \multicolumn{1}{|c|}{ Scintillators } & \multicolumn{1}{c|}{ Remarks } \\
\hline 1 & ZnS:Ag & $\lambda_{\max }=450 \mathrm{~nm}[10]$ \\
\hline 2 & YAG:Ce & $\lambda_{\max }=550 \mathrm{~nm}[7]$ \\
\hline 3 & E6 Ib (single crystal) & No NV \\
\hline 4 & E6 EL IIa (single crystal) & No NV \\
\hline 5 & Polycrystalline & No NV \\
\hline 6 & HPHT IIa (single crystal) & Contains NVs \\
\hline
\end{tabular}

particles are adhered to a plastic sheet by using an organic resin adhesive. The thicknesses of the $\mathrm{ZnS}: \mathrm{Ag}$ and plastic layers are 20 and $15 \mu \mathrm{m}$. When $\mathrm{ZnS}: \mathrm{Ag}$ is placed on a semiconductor, the plastic sheet is removed. The next scintillator considered is a YAG:Ce (\#2), which is considered to be the most promising scintillator for IPEM [7]. The thickness of YAG:Ce is $0.2 \mathrm{~mm}$. The emission maximum is at $550 \mathrm{~nm}$ and the PL spectrum completely matches the QEs of the detectors. Finally, various diamonds are used in this study. Three different varieties of diamonds without NV centers are studied: first is a Ib single crystal diamond purchased from Element Six (E6) (\#3); second is an electronic grade IIa single crystal diamond purchased from E6 (\#4); third is a polycrystalline diamond synthesized at National Institute for Materials Science (NIMS) (\#5). These samples do not include NV centers. In addition, a non-commercial HPHT (High Presser High Temperature) IIa single crystal diamond containing NV centers (\#6) is studied. The NV centers are created by high energy electron irradiation and annealing. The distribution of NV centers is uniform and its concentration is $0.14 \mathrm{ppm}\left(2.5 \times 10^{16} \mathrm{~cm}^{-3}\right)$. The PL spectrum of \#6 measured at NIMS is shown in Fig. 2 (b). As mentioned in introduction, the Zero Phonon Lines (ZPLs) of $\mathrm{NV}^{0}$ and $\mathrm{NV}^{-}$are found at the wavelengths of 575 and $637 \mathrm{~nm}$. The broad spectrum $(550-800 \mathrm{~nm})$ corresponds to vibronic side bands of each charge state. 
The QE of the I.I. decreases at the emission maximum of NV centers in the diamond. Since there is partial overlap between the QEs of the detectors and the PL spectrum of NV centers in the diamond, the portion of the emitted photons from NV centers in the diamond can be detected.

\section{RESULTS AND DISCUSSION}

\subsection{Single Ion Detection by Using IPEM}

Fig. 3 (a) shows the micrograph of the scintillator (ZnS:Ag) on a silicon (Si) diode. During a $150 \mathrm{MeV}$ Ar ion irradiation, the CCD image is captured for $20 \mathrm{~ms}$, as show in Fig. 3 (b). Nine spots are detected, and each spot can be distinguished. Applying the beam attenuator to control the beam flux, the number of spots per one frame can be controlled. It is confirmed that the number of spots agrees with the number of signals detected by the semiconductor device (solid state detector) under the scintillator.

The position where the ion hit the sample is calculated from the center of mass of each spot. Although not shown here, we measure the ion luminescence from the $\mathrm{ZnS}: \mathrm{Ag}$ covered with Cupper mesh with the gap of about $250 \mu \mathrm{m}$. Since the projected range of ion is shorter than the thickness of mesh, the ion luminescence is detected when ions pass through the gap of mesh. By calculating the center of mass of each spot, the image of mesh is successfully observed.
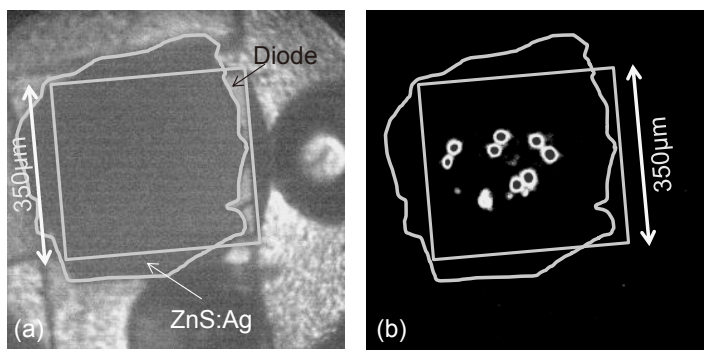

Fig. 3 Micrograph of the $\mathrm{ZnS}: \mathrm{Ag}$ on a Si diode (a) and the image of CCD when the $150 \mathrm{MeV}$ Ar ions pass through the $\mathrm{ZnS}: \mathrm{Ag}(\mathrm{b})$.
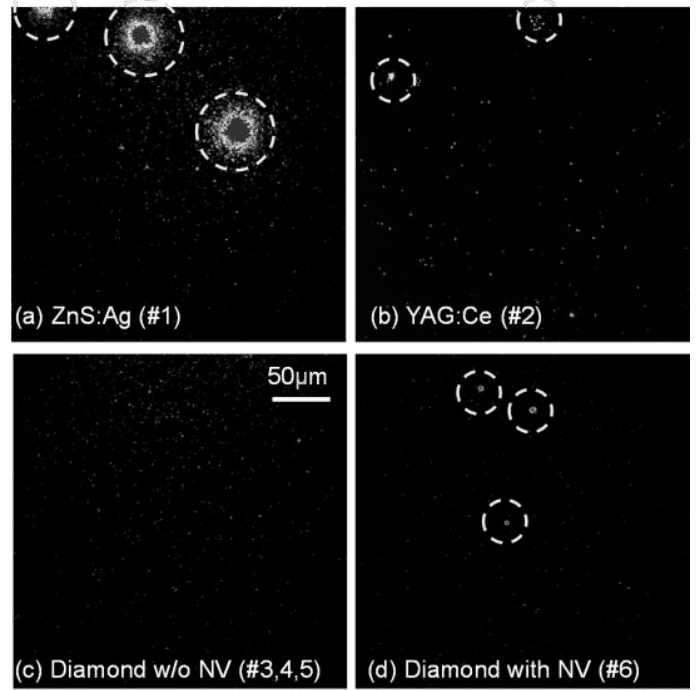

Fig. $4 \mathrm{CCD}$ images when the $150 \mathrm{MeV}$ Ar ions penetrate the ZnS:Ag (a), the YAG:Ce (b), diamonds without NV centers (c), and the diamond containing NV centers (d).
Fig. 4 shows the CCD images when $150 \mathrm{MeV}$ Ar ions penetrate various scintillators. The dashed circles highlight the ion induced spots. The Ion Beam Induced Luminescence (IBIL) is observed from $\mathrm{ZnS}: \mathrm{Ag}$, YAG:Ce and the diamond containing NV centers, as shown in Fig. 4 (a, b, d), respectively. But no spot is observed from diamonds without NV centers (\#3, \#4, \#5) as shown in Fig. 4 (c). This fact suggests that the NV center is the main contributor to the IBIL image. One possibility of the luminescence mechanism from a diamond containing NV centers is following. According to the ion track distribution theories, the radius of ion track created by the single ion with the several hundreds of $\mathrm{MeV}$ exceeds $1 \mu \mathrm{m}$ [11]. Since a lot of NV centers distribute in the ion track, these are excited. When the excited NV centers change to the ground state, the IBIL is emitted. To confirm this, it is necessary to measure the IBIL spectrum. Therefore it needs further consideration.

As shown in Fig. 4 (a, b, d), the spot sizes in YAG:Ce and diamond are much smaller than that of $\mathrm{ZnS}: \mathrm{Ag}$. The spot size of $\mathrm{ZnS}: \mathrm{Ag}$ corresponds to the diameter of $\mathrm{ZnS}$ :Ag powders. The spot size in an IPEM scintillator is one of the most important properties, since it determines the spatial resolution. Fig. 5 (a) shows the averaged spot distribution observed from \#1, \#2 and \#6. The dashed line shows the noise level. The Signal to Noise $(\mathrm{S} / \mathrm{N})$ ratios of each scintillator are $7.7,3.4$ and 4.6, respectively. The Full Width at Half Maximums (FWHMs) of spot are evaluated to be 16, 2.9 and $3.8 \mu \mathrm{m}$, respectively. Fig. 5 (b) shows the IBIL intensity normalized by the peak value. The spot distribution of YAG:Ce (\#2) agrees well with that of the diamond (\#6). In contrast with our data, it is reported that the spatial resolution of about $5 \mu \mathrm{m}$ has been achieved by using YAG:Ce [7]. In the case of n-type Gallium Nitride (n-GaN), the spatial resolution of about $2.5 \mu \mathrm{m}$ has been demonstrated [6]. Therefore, we suggest that a diamond containing NV centers is a rival candidate of YAG:Ce and $\mathrm{n}-\mathrm{GaN}$ in spatial resolution.

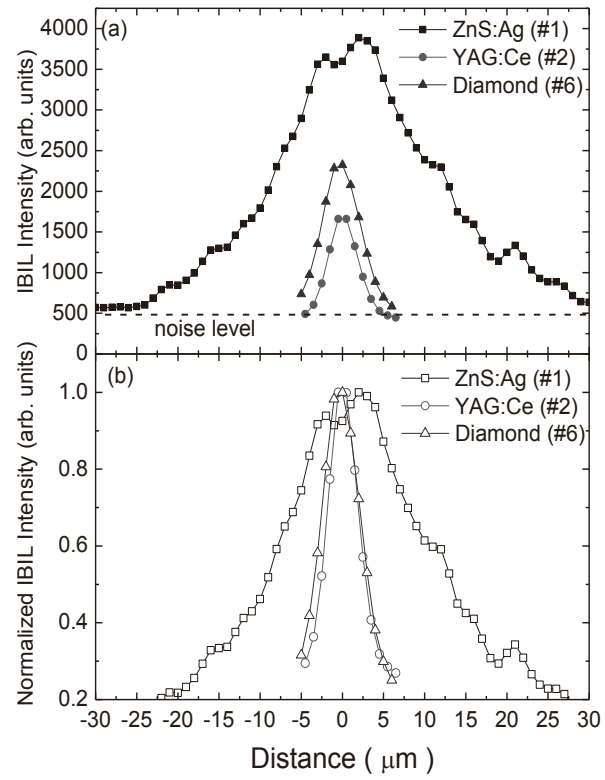

Fig. 5 Averaged IBIL intensity as a function of distance across each spot from Fig. 4 (a). The normalized IBIL is represented in (b). The IBIL is observed after the 150 $\mathrm{MeV}$ Ar ion strikes. 


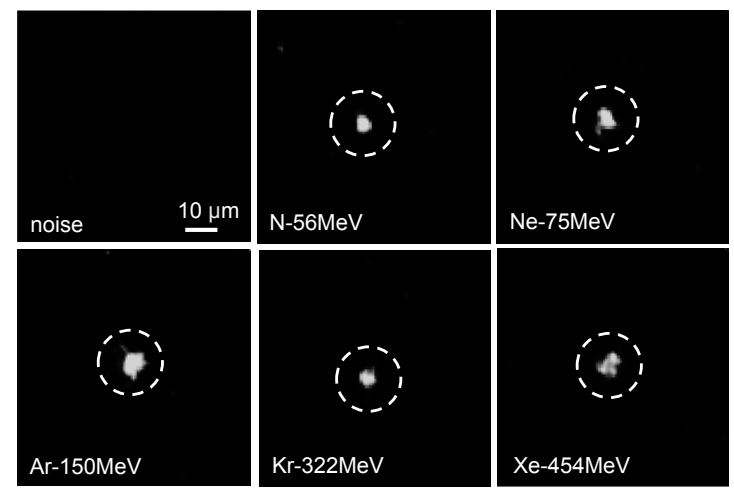

Fig. $6 \mathrm{CCD}$ images when various ions with various energies penetrate the diamond (\#6).

The emission rate is also an important property. Here we will use the term "emission rate" to refer to the number of emitted photons from the scintillator. To evaluate the emission rate, we calculate the integrated values of measured IBIL spectra shown in Fig. 5 (a). The integrated values for $\# 1, \# 2$ and $\# 6$ are observed to be 81752,2783 and 11288 , respectively. In this calculation, the IBIL spectra over noise level are integrated. The integrated value of IBIL from YAG:Ce $(\# 2)$ is about 30 times smaller than that from $\mathrm{ZnS}: \mathrm{Ag}$ (\#1), and 4 times smaller than that from diamond (\#6). On the other hand, as previously mentioned, the QEs of detectors completely overlap the PL spectra of $\mathrm{ZnS}: \mathrm{Ag}$ and YAG:Ce. However, the QE of the I.I. decreases at the emission maximum of NV centers in the diamond. In other words, despite the mismatch between its emission maximum and QE of the I.I., the integrated values of IBIL from the diamond (\#6) is 4 times higher than that from the YAG:Ce (\#2). Since the integrated value depends on the emission rate and QEs of the detectors, the diamond containing NV centers emits the photons far more than the YAG:Ce. To utilize a diamond containing NV centers as a scintillator for IPEM, it is better to use other photocathodes sensitive to wavelengths up to $800 \mathrm{~nm}$ witch math, for example a $\mathrm{GaAs}$ photocathode with sensitive wavelength ranging from 400 to $900 \mathrm{~nm}$.

\subsection{Ion Species and Energy Dependence}

It is well known that the Single Event Effects (SEEs) in microelectronics depend on the ion species and energy. In this study, we evaluate the ability of the diamond containing NV centers as a scintillator for IPEM. The Nitrogen (N), Neon (Ne), Argon (Ar), Krypton $(\mathrm{Kr})$ and Xenon (Xe) with the energies of 56, $75,150,322$ and $454 \mathrm{MeV}$, are irradiated to the diamond containing NV centers. Typical CCD images are shown in Fig. 6. The dashed circles highlight the ion induced spots. It is found that the spots are detected for all cases. In this study we successfully demonstrate that the diamond containing NV centers can be used to detect a wide variety of ion species and energies. It is debatable whether the spot size and intensity of IBIL depends on the ion species and energies.

\section{SUMMARY}

To achieve high spatial resolution mapping of Single Event Effects (SEEs) by using Ion Photon Emission
Microscopy (IPEM) system, the exploration of optimum ion luminescent materials is required. It has been reported that YAG:Ce demonstrates the most promising properties [7].

As a result of ion irradiation, the IBIL is observed from the YAG:Ce and the diamond containing NV centers. No IBIL is observed from diamonds without NV centers. This fact suggests that NV centers mainly contribute to the IBIL. We found that the spot size of the diamond containing NV centers is comparable with the YAG:Ce after normalization. In addition, it is found that the luminescence intensity of the diamond containing NV centers is higher than that from YAG:Ce. The S/N ratio of the diamond containing NV centers is better than that of the YAG:Ce. To detect a single ion with high sensitivity, it is better to use the diamond containing NV centers instead of YAG:Ce. Finally, we demonstrate that the diamond containing NV centers can be used to detect the single ion with a wide variety of ion species and energies. According to these results, we therefore conclude that a diamond containing NV centers is a rival candidate of a YAG:Ce for IPEM scintillator applications.

\section{ACKNOWLEDGMENT}

The YAG:Ce was provided by Mr. A. Yokoyama of the Department of Advanced Radiation Technology, JAEA. This work was partly supported by the Strategic Japanese-German Joint Research Program in 2009 from the Japan Science and Technology Agency.

\section{REFERENCES}

[1] I. Nashiyama, T. Hirao, T. Kamiya, H. Yutoh, T. Nishijima and H. Sekiguti, IEEE Trans. Nucl. Sci., 40, 1935-1940 (1993).

[2] J. S. Laird, T. Hirao, H. Mori, S. Onoda, T. Kamiya and H. Itoh, Nucl. Instr. And Meth. B, 181, 87-94 (2001).

[3] T. Hirao, S. Onoda, M. Oikawa, T. Satoh, T. Kamiya and T. Ohshima, Nucl. Instr. And Meth. B, 267, 2216-2218 (2009).

[4] H. Schone, D. S. Walsh, F. W. Sexton, B. L. Doyle, P. E. Dodd, J. F. Aurand, R. S. Flores and N. Wing, IEEE Trans. Nucl. Sci., 45, 2544-2549 (1998).

[5] B. L. Doyle, D. S. Walsh, S. N. Renfrow, G. Vizkelethy, T. Schenkel and A. V. Hamza, Nucl. Instr. And Meth. B, 181, 199-210 (2001).

[6] J. V. Branson, B. L. Doyle, G. Vizkelethy, P. Rossi, J. A. Knapp and M. A. McMahan, Nucl. Instr. And Meth. B, 267, 2085-2089, (2009).

[7] J. V. Branson, K. Hattar, P. Rossi, G. Vizkelethy, C. J. Powell, B. Hernandez-Sanchez and B. L. Doyle, Nucl. Instr. And Meth. B, 269, 2326-2329 (2011).

[8] A. M. Zaitsev, "optical properties of a diamond: A Data Handbook", Springer (1998).

[9] I. Aharonovich, S. Castelletto, D. A. Simpson, C-H. Su, A. D. Greentree, and S. Prawer, Rep. Prog. Phys., 74, 076501 (28pp) (2011).

[10] S. K. Lee, S. Y. Kang, D. Y. Jang, C. H. Lee, S. M. Kang, B. H. Kang, W. G. Lee and Y. K. Kim, Prog. Nucl. Sci. and Tech., 47, 194-197 (2011).

[11] O. Fageeha, J. Howard, and R. C. Block, Jour. Appl. Phys., 75, 2317-2321 (1994).

(Received January 20, 2012; Accepted May 7, 2012) 